\title{
Comparison of fine needle aspiration cytology, radioisotopic and ultrasound scanning in the management of thyroid nodules
}

\author{
A.J. Jones, T.J. Aitman, C.J. Edmonds, M. Burke, E. Hudson and M. Tellez \\ Endocrine Research Group and Section of Histopathology, Clinical Research Centre, Northwick Park \\ Hospital, Watford Road, Harrow, Middlesex HAl 3UJ, UK
}

Summary: In 175 patients presenting with thyroid nodules, the diagnostic value in management of fine needle aspiration and cytology (FNAC), pertechnetate ${ }^{99 m}$ Tc) scanning and ultrasound imaging was examined. In 82 patients, the diagnosis was confirmed at operation; in the remaining 93 , there was a follow-up period of at least 2 years. Thyroid cancer was found in 13 patients. For FNAC the sensitivity, specificity and positive predictive value for thyroid cancer were $92 \%, 85 \%$ and $41 \%$ respectively compared with $82 \%, 34 \%$ and $11 \%$ for ${ }^{99 m}$ Tc pertechnetate scanning, $75 \%, 61 \%, 19 \%$ for ultrasound and $73 \%, 58 \%$ and $19 \%$ for combined pertechnetate and ultrasound scanning. In $14 \%$ of patients, the aspirates were inadequate for cytology at the first examination.

FNAC is therefore the preferable initial investigation and usually gives results adequate for a decision on surgical or medical management. With medical management and follow-up, ultrasound is of value in defining the nodule and the appearance of the rest of the gland.

\section{Introduction}

Euthyroid patients may present with what appears to be a single nodule or with multinodular goitre having a dominant nodule which excites suspicion because of its size or because it is thought to be enlarging. The investigation of this common clinical problem and the selection of those patients who need surgery because of the possibility of thyroid cancer, has been the subject of much discussion.

Three main modalities of investigation are available, namely, isotopic thyroid scanning, ultrasound scanning and fine needle aspiration cytology (FNAC). A number of studies have advocated FNAC as the best or even the sole investigation, ${ }^{1-4}$ but there have been few comparative studies along the lines accepted for evaluation of diagnostic tests $^{5,6}$ and expensive scanning procedures have continued to be widely used. Since subsequent management, either surgical or medical, depends on the results of these investigations it is of importance that their comparative value should be assessed. Over several years, the great majority of our patients presenting for investigation of thyroid nodules have been investigated by all three methods and have, moreover, been followed up for some years subsequently. This has enabled us to examine, in the setting of a district general hospital, the value of these tests in identifying thyroid cancer and the need for surgical removal of the lesion.

Correspondence: C.J. Edmonds, M.D., D.Sc., F.R.C.P. Accepted: 9 July 1990

\section{Materials and methods}

All euthyroid patients who were investigated by FNAC of the thyroid during the $4 \frac{1}{2}$ year period September 1983 to March 1988 with subsequent partial thyroidectomy (82 patients) and all patients who had FNAC during the 4 year period September 1983 to September 1987 with subsequent 2 year follow-up but no operation ( 93 patients) were included in the study. These patients were seen at 6 monthly intervals with ultrasound examination yearly. The decision for or against partial thyroidectomy in some patients was made by the clinicians in charge on the basis of their clinical assessment and the results of all the investigations. Of the 93 patients who had no operation, 19 had a simple thyroid cyst which was adequately treated by the aspiration with complete disappearance of the thyroid mass. ${ }^{7}$ Other investigations at presentation always included thyroid function tests and antibody titres.

Aspiration for cytology was mainly performed by one of us (CJE). For aspiration, the neck was extended, supported by a small pillow and a $10 \mathrm{ml}$ syringe with a 23 gauge needle was used. Two or three separate aspirations were made with several passes through the lesion. The aspirates were air-dried on microscope slides and stained with May-Greenwald-Giemsa stain. Throughout the study period, the aspirates were reported by one cytologist (EH). Thyroid radioisotope scanning 
was done using Scintag gamma camera and a pinhole collimator at 20 minutes after an intravenous bolus of $80 \mathrm{MBq}$ of pertechnetate ${ }^{99_{\mathrm{m}} \mathrm{Tc}}$. Ultrasound scanning was done using a $7.5 \mathrm{MHz}$ mechanical sector transducer and direct skin contact.

The reports for the various investigations were reviewed by one of us (AJJ) and scored as positive (i.e. consistent with the lesion being thyroid cancer) if (a) the cytology report said the appearances were those of cancer or an adenoma or had suspicious cytological appearance, (b) if the ultrasound report said the lesion was solid or a mixed solid, cystic structure, (c) if pertechnetate scan showed the lesion was nonconcentrating, that is 'cold'.

In the analysis of the results, the terms sensitivity, specificity and positive predictive value have their usual meaning. ${ }^{5,6}$

\section{Results}

The performance of FNAC in establishing a morphological diagnosis in the 82 patients proceeding to thyroidectomy is shown in Table I. Of those 93 patients who did not undergo thyroidectomy, one had a thyroid lymphoma; in the remaining patients, a minimum of 2 years of follow-up has been completed and no malignancies have emerged. In 19 of the 175 patients, the nodule was shown to be a simple cyst by ultrasound and on fine needle aspiration of the fluid, the nodule disappeared. Thyroid cancer was found in 13 of the patients, that is, in about $7 \%$ of the whole series and in $9 \%$ of those with solid nodules.

One patient, a man of 55 years in the thyroidectomy group, had a false negative FNAC report (that is a benign-cytology report in a carcinoma of the thyroid), giving a false negative rate of $8 \%$. The thyroid aspirate of this patient contained a small amount of colloid and several round clusters of follicle cells. It was reported as a colloid nodule provided that the aspirate was a representative sample. At thyroidectomy a well differentiated follicular carcinoma was removed: histology showed small acini and some colloid.

In 24 patients $(14 \%)$ the specimens were considered to be inadequate but just over $60 \%$ of these were taken during the first 2 years of the study suggesting that the number of inadequate aspirations decreases with experience. Table II shows the sensitivity, specificity and predictive value of FNAC isotope and ultrasound scanning for detection of malignancy. Although both pertechnetate and ultrasound scanning scored well for sensitivity, both techniques were relatively nonspecific. Pertechnetate scanning was poorer in this respect than ultrasound as it failed to distinguish between cystic and solid lesions. Cytology was more specific but in one patient the cancer was not recognized. In this

Table I Cytological reports compared with the histology of tissues obtained at thyroidectomy in 82 patients

\begin{tabular}{lccccc}
\hline & \multicolumn{2}{c}{ Histology } \\
Cytology & $\begin{array}{c}\text { Papillary follicular } \\
\text { cancer }\end{array}$ & Adenoma & Thyroiditis $\begin{array}{c}\text { Benign } \\
\text { Colloid nodule } \\
\text { or MNG }\end{array}$ \\
\hline Cancer & 4 & 2 & 0 & 0 & 1 \\
Possibly & 3 & 1 & 7 & 1 & 10 \\
Benign & 0 & 1 & 8 & 4 & 23 \\
Inadequate & 0 & 1 & 3 & 0 & 13 \\
\hline
\end{tabular}

MNG, multinodular goitre

Table II Evaluation of diagnostic tests for cancer in thyroid nodules

\begin{tabular}{|c|c|c|c|c|}
\hline & Cytology & Ultrasound scan & Pertechnetate scan & $\begin{array}{c}\text { Ultrasound and } \\
\text { pertechnetate scans }\end{array}$ \\
\hline Sensitivity (\%) & 92 & 75 & 82 & 73 \\
\hline Specificity (\%) & 85 & 61 & 34 & 58 \\
\hline $\begin{array}{l}\text { Positive }(\%) \\
\text { predictive } \\
\text { value }\end{array}$ & 41 & 19 & 11 & 19 \\
\hline
\end{tabular}

All 175 patients had FNAC; 141 patients had both ultrasound and pertechnetate scans, the remaining 34 having either ultrasound or pertechnetate scan. 
patient, a short history of an enlarging nodule in the thyroid of a middle aged man together with ultrasound demonstration of a solitary solid nodule in an otherwise normal gland led to thyroidectomy being undertaken.

\section{Discussion}

FNAC can often suggest a precise diagnosis of a thyroid lesion but, from the point of view of management, the important issue is whether the findings suggest cancer. The present results show that FNAC is the investigation of highest specificity and positive predictive value. However, even though the number of resections is substantially reduced when FNAC is used to guide management ${ }^{8,9}$ some unnecessary operations are still performed because of false positive reports.

The possibility of the false negative has always to be borne in mind for it has averaged about $5-14 \%$ in most studies. ${ }^{1,8,10,11}$ The one false negative result in our series illustrates the problem arising because a follicular carcinoma may not be distinguishable from a very cellular colloid nodule or a benign adenoma. If the tumour contains much colloid, which may ocasionally happen in follicular cancers, then on cytological evidence alone it may be regarded as a colloid nodule. This was so in the one patient of our series whose thyroid cancer appeared cytologically negative. In deciding on management, therefore, the history is important and ultrasound is valuable to determine if the lesion is solitary and as an objective indication of the size of the lesion during follow-up. Combining ultrasound and FNAC in all patients as the initial assessment ${ }^{12}$ is unnecessary although after review of the cytology, ultrasound is of value in those patients in whom follow-up without surgery is contemplated. Enlarging isolated lesions in an otherwise normal gland are best removed.

Inadequate specimens are also fairly frequent, usually at a rate of about $20 \%$ for a single examination but this can be reduced by repeating the procedure. ${ }^{3,8,13}$ Increasing experience of the operator and cytologist is also important but the present observations indicate that satisfactory

\section{References}

1. Van Herle, A.J., Rich, P., Ljung, B.-M.E., Ashcraft, M.A., Solomon, D.N. \& Keeler, E.B. The thyroid nodule. Ann Intern Med 1982, 96: 221-232.

2. Blum, $\mathbf{M}$. The diagnosis of the thyroid nodule using aspiration biopsy and cytology. Arch Intern Med 1984, 144: 1140-1142.

3. Franklyn, J.A., Fitzgerald, M.G., Oates, G.D. \& Sheppard, M.C. Fine needle aspiration cytology in the management of euthyroid goitre. $Q J$ Med 1987, 248: 997-1003. results can be achieved by the modest experience (from about 40 new cases a year) obtained from the workload of a single general hospital.

These limitations are offset by the considerable advantages of FNAC. Its discriminating value is relatively high compared with the scanning methods and it is much less consuming of time and expensive equipment. Moreover if the nodule proves to be a simple cyst, the diagnostic procedure can be combined with that of treatment, namely cyst aspiration, and, if necessary, sclerosant injection which is usually curative.?

A simple protocol for management of the euthyroid patient with a thyroid nodule is shown in Figure 1. This places FNAC as the initial investigation (with thyroid function tests) at the first clinical visit. If this protocol had been used in the present series of patients, surgery would have been avoided in at least 36 patients (all of whom had benign lesions on cytology) of the 82 who underwent thyroidectomy. If medical management is elected, then ultrasound examination is done to secure an objective record of the nodule and the thyroid; it may also reveal multinodularity not recognized clinically. The pertechnetate scan is probably useful only if thyroid function tests suggest hyperactivity (e.g. suppressed serum TSH) when a 'hot' nodule may be demonstrated.

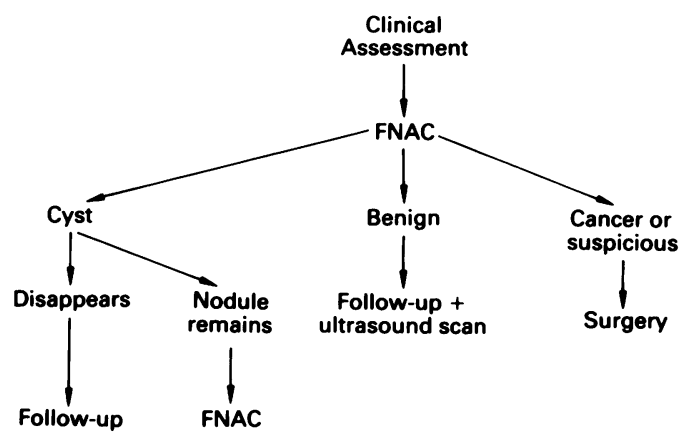

Figure 1 Protocol for management of a thyroid nodule in a euthyroid patient. (Surgery may also be required by the patient for cosmetic reasons.)

4. Smeds, S. \& Lennquist, S. The role of aspiration cytology in the management of thyroid nodules. Eur J Cancer Clin Oncol 1988, 24: 293-297.

5. Griner, P.F., Mayewski, R.J., Mushlin, A.I. \& Greenland, P. Selection and interpretation of diagnostic tests and procedures. Ann Intern Med 1981, 94: 553-592.

6. Sheps, S.B. \& Schechter, M.T. The assessment of diagnostic tests. A survey of current medical research. JAMA 1984, 252: 2418-2422. 
7. Edmonds, C.J. \& Tellez, M. Treatment of thyroid cysts by aspiration and injection of sclerosant. Br Med J 1987, 295: 529.

8. Anderson, J.B. \& Webb, A.J. Fine-needle aspiration biopsy and the diagnosis of thyroid cancer. $B r J$ Surg 1987, 74: 292-296.

9. Kendall, C.H. Fine needle aspiration of thyroid nodules: three years experience. J Clin Pathol 1989, 42: 23-27.

10. Hawkins, F., Bellido, D., Bernal, C. et al. Fine needle aspiration biopsy in the diagnosis of thyroid cancer and thyroid disease. Cancer 1987, 59: 1206-1209.
11. Mazzaferri, E.L., de los Santos, E.T. \& Rofagha-Keyhani, S. Solitary thyroid nodule: diagnosis and management. Med Clin North Am 1988, 72: 1177-1211.

12. Wallfish, P.G., Hazani, E., Strawbridge, H.T.G., Miskin, M \& Rosen, I.B. Combined ultrasound and needle aspiration cytology in the assessment and management of hypofunctioning thyroid nodule. Ann Intern Med 1977, 87: 270-274.

13. Ramacciotti, C.E., Pretorius, H.T., Chu, E.W., Barsky, S.H., Brennan, M.F. \& Robbins, J. Diagnostic accuracy and use of aspiration biopsy in the management of thyroid nodules. Arch Intern Med 1984, 144: 1169-1173. 